\title{
Feasibility and Benefits of Passive RFID Wake-up Radios for Wireless Sensor Networks
}

\author{
He Ba, Ilker Demirkol, and Wendi Heinzelman \\ Department of Electrical and Computer Engineering \\ University of Rochester, Rochester, NY, USA \\ e-mail:\{ba,demirkol,wheinzel\}@ece.rochester.edu
}

\begin{abstract}
Energy efficiency is one of the crucial design criteria for wireless sensor networks. Idle listening constitutes a major part of energy waste, and thus solutions such as duty cycling and the use of wake-up radios have been proposed to reduce idle listening and save energy. Compared to duty cycling, wake-up radios save more energy by reducing unnecessary wake-ups and collisions. In this paper, we investigate the feasibility and potential benefits of using passive RFID as a wake-up radio. We first introduce a physical implementation of sensor nodes with passive RFID wake-up radios and measure their energy cost and wake-up probability. Then, we compare the performance of our RFID wake-up sensor nodes with duty cycling in a Data MULE scenario through simulations with realistic application parameters. The results show that using a passive RFID wake-up radio offers significant energy efficiency benefits at the expense of delay and the additional low-cost RFID hardware, making RFID wake-up radios beneficial for many delaytolerant sensor network applications.
\end{abstract}

Keywords-Wireless sensor networks; wake-up receivers; passive RFID wake-up; data MULE

\section{INTRODUCTION}

Wireless sensor networks (WSNs) consist of a number of sensor nodes that have the ability to sense the environment, process the sensed data, and disseminate the processed data to one or more sinks. WSNs have been proposed for several applications including disaster monitoring, surveillance, target tracking, and health monitoring. Since the sensor nodes are usually powered by batteries, WSNs are highly energy constrained, creating the need for innovative solutions to reduce energy dissipation.

Idle listening, when a sensor node is active and waiting to receive data, is a large source of energy drain in WSNs. Generally there are two approaches to reduce the energy consumption due to idle listening: duty cycling the node and using a wake-up radio. Since sensor nodes do not have data to send all the time, it is common to use duty cycling, where the nodes are periodically set into the sleep mode. Duty cycling saves a significant amount of energy at the expense of latency in data delivery. However, one problem in utilizing duty cycling is that the nodes wake up periodically regardless of whether or not any other nodes have data to transmit to them. In this situation, the nodes waste significant energy due to unnecessary wake-ups. Furthermore, duty cycling relies on tight time synchronization among the nodes to achieve good performance. On the other hand, when utilizing wake-up radios, the nodes are awakened by neighboring nodes only when they need to receive packets. Such an on-demand mechanism has the potential to save significant energy waste due to idle listening, unnecessary wake-ups, overhead in control traffic, and collisions.

Wake-up radios can be classified into two categories as active and passive wake-up radios. Active wake-up radios consume power, but they have longer wake-up ranges than passive wake-up radios. Passive wake-up radios use the energy harvested from the wake-up radio and thus operate over short ranges. One possibility is to use passive RFID as the wake-up technology, as there are off-the-shelf passive RFID tags and readers readily available. A major drawback of using passive RFID tags for the wake-up functionality is that multi-hop communications cannot be supported due to the large size and large power consumption of the RFID reader. It is not yet practical to equip all sensor nodes with RFID readers. Additionally, it is not known how well passive RFID would perform as a wake-up radio, in terms of wake-up distance, wake-up probability, and energy consumption for the sensor node to be woken up. Hence, determining the feasibility of using passive RFID for a wake-up radio and the potential benefits of such a wake-up radio in real scenarios require a separate study, which is the aim of this paper.

In this paper, we describe a physical implementation of a passive RFID wake-up device using existing hardware. By combing WISP (Wireless Identification and Sensing Platform) passive RFID tags developed by Intel Research [1] with Tmote Sky motes [2], we created a passive RFID wake-up device, which is referred to as a WISP-Mote in this paper. We characterize the performance of the WISP-Motes by measuring the power consumption in different operation stages, including sleeping, wake-up, transmitting and receiving, and by testing the wake-up probability for different ranges. To show the benefits of WISP-Motes, and hence the benefits of passive RFID-based wake-up radios, we compare the use of WISPMotes with a standard mote architecture that utilizes duty cycling for a single-hop Data MULE [3] data collection scenario. 
The remainder of this paper is organized as follows. In Section II, we discuss the related work on wake-up radio architectures and the use of data MULEs in WSNs. Section III presents the implementation and characterization of our passive RFID wake-up mote, the WISP-Mote. A comparison of the performance of the WISP-Mote and the duty-cycle architectures for single-hop data MULE scenarios are provided in Section IV. Section V concludes the paper.

\section{RELATED WORK}

Wake-up radios can be categorized as active or passive. A passive radio wake-up circuit does not consume any energy from the batteries, while an active one requires a power supply. Different low-power radio wake-up receivers have been designed, such as those described in [4-6]. Gu et al. proposed a passive radio wake-up circuit that theoretically could operate at a range of 10 feet with $5 \mathrm{~ms}$ latency based on SPICE simulation results [7]. If a comparator and an amplifier, which respectively consume negligible currents of $350 \mathrm{nA}$ and $880 \mathrm{nA}$, are added to the wake-up circuit, it could theoretically reach up to 100 feet with $55 \mathrm{~ms}$ latency. However, there are no existing physical implementations of passive RFID wake-up radios described in the literature.

To the best of our knowledge, the only performance study on the passive RFID wake-up technique is by Jurdak et al. [8], [9]. In their work, an RFID wake-up mechanism is proposed, namely RFIDImpulse, for which analytical models of energy consumption are presented. The performance of the proposed mechanism was investigated through MATLAB simulations and compared with BMAC and the IEEE 802.15.4 standard. Their results show that RFIDImpulse performs better than both of the other methods for low and medium traffic scenarios. However, an important assumption is that all nodes have the capability to wake up their neighbors, which is not feasible in real scenarios, due to the considerable amount of energy consumed by the RFID reader as well as its large size. In addition, the energy consumption analysis does not include the energy consumed by the nodes to wake up. In reality, the wakeup energy consumption includes the energy used for MCU boot-up and for radio initiation, which can be comparable to the energy consumed for radio transmission. In our work, the energy consumed during wake-up is also considered and is based on the actual measurements for our WISP-Mote device.

To have wide network coverage, WISP-Motes are used with mobile sinks in this paper due to their short wake-up ranges. The mobile sink wakes up the WISP-Motes when it gets within their wake-up range to collect their data. This is similar to the three-tier layered architecture described in [10], where a simple analytical model for a data MULE network is presented based on random walks on a two-dimensional grid. The grid is used to simplify the communication by assuming the transmission is successfully completed when the MULE and the sensor are in the same grid point. In our scenarios, we investigate random walk along with two other MULE mobility models and present their performance comparison.

In [11], a source-to-sink delay analysis in a single-hop mobile sink scenario is presented. The nodes are placed in a rectangular area with a mobile sink moving along the central axis of a rectangular area. The authors assume a negligible transmitting and queuing delay. The simulation results show that the average delay decreased as the transmission radius increased and as the mobile sink velocity increased. A MULE discovery protocol is presented in [12] where the data MULE periodically advertises its presence by sending special messages called beacons. The static nodes periodically wake up, and once a node receives a complete beacon, it switches to the always-on mode and sends its data. After transferring all available data, the node goes back to its periodic wake up scheme. Simulation results show that this protocol achieves energy efficiency with a low duty cycle, while achieving a throughput sufficient for common environmental monitoring. We employ a similar beacon-based wake-up mechanism in our simulations for duty cycling scenarios.

\section{THE WISP-MOTE PLATFORM}

The lifetime of a wireless sensor network node is limited by the sensor node's battery supply. To extend a node's lifetime, duty cycling can be utilized. To reduce the node's energy consumption, the duty cycle must be set to a relatively low value (e.g., $10 \%$ duty cycle, which means the node is "on" for $10 \%$ of the time). However, this will increase the average data transmission latency, as packets that arrive at a node during the sleep period must be buffered until the next active period. Although there are protocols designed to reduce large delays caused by sleeping, such as DMAC [13], these approaches require additional overhead and global routing management. When a node has no information about its environment, idle listening is inevitable with the duty cycling approach. Besides idle listening, control packet overhead and synchronization overhead are also sources of energy waste observed with duty cycle approaches. All of the above issues motivate us to utilize radio wake-up techniques in wireless sensor networks to further improve energy efficiency. This section introduces the implementation of a combined passive RFID-based wake up radio and a sensor mote, which we call a WISP-Mote, and provides measurement results of the wake-up probability and the energy consumption of the WISP-Motes.

\section{A. Radio Wake-up Basics}

Most sensor nodes use a microcontroller (MCU) to provide computation and data processing, control the radio and sensors, and manage memory and power. An internal clock, called the watchdog timer, is used to wake up the system when a timer fires. By setting this timer, a node can wake up periodically to perform its functionalities. On the other hand, nodes lose their functionalities while sleeping. The only other way to wake up a node from the sleep state is to send an external interrupt signal through the pins of the MCU. Such an external interrupt signal is generated by the radio wake-up circuitry.

\section{B. Implementation}

A WISP is a passive RFID tag with simple sensing and computing capabilities, developed by Intel Research for research purposes. A WISP can be powered and read by an offthe-shelf UHF RFID reader. Tmote Sky motes are widely used sensor nodes. Both devices' specifications are presented in Table I. 
TABLE I. DEVICE SPECIFICATIONS

\begin{tabular}{|l|l|}
\hline \multicolumn{1}{|c|}{ WISP 4.1DL [14] } & \multicolumn{1}{|c|}{ Tmote Sky [15] } \\
\hline $\begin{array}{l}\text { TI MSP430 F2132 (512B } \\
\text { RAM, 8K+256B Flash) }\end{array}$ & TI MSP430 F1611 (10k RAM, 48k Flash) \\
\hline & $\begin{array}{l}\text { 250kbps 2.4GHz IEEE 802.15.4 Chipcon } \\
\text { Wireless Transceiver }\end{array}$ \\
\hline Accelerometer, Temp. & Humidity, Temperature, Light \\
\hline & Fast wakeup from sleep (<6 $\mu \mathrm{s}$, typically 292ns) \\
\hline
\end{tabular}

We combine a WISP and a Tmote Sky (mote) to create a passive wake-up mote, a WISP-Mote, as shown in Fig. 1. We wire an output pin of the WISP to one of the mote's GPIO pins to send an interrupt signal. The voltage required to trigger an external interrupt is $0.92 \mathrm{~V}$. The output signal from the WISP's MCU is $1.8 \mathrm{~V}$, which is enough to wake up the mote. To make our WISP-Mote stable, we also need to wire the GNDs of the two devices together.

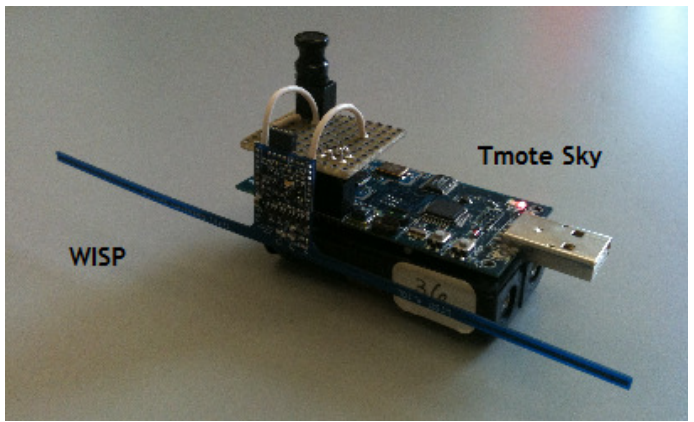

Figure 1. A WISP-Mote.

There were a few challenges faced in creating a robust WISP-Mote. These include:

- The ubiquitous noise in and between the WISP and the mote is large enough to influence the wake-up. To avoid this, we bridge the two GNDs with a large inductor to filter high frequency noise.

- The communication range of the WISP is limited, less than 3 meters, if it runs the UHF RFID standard C1G2 protocol. In order to extend the wake-up range, we disable the WISP-to-reader communication and eliminate all other computation burdens in the WISP MCU. The only function of the WISP is to keep harvesting power from the reader's radio and output an impulse once it can. Using this approach, the wake-up range is extended to approximately 5 meters as shown in this paper.

\section{Wake-up Probability}

The energy a WISP is able to harvest decreases with increasing reader-to-WISP distance due to path loss. Thus, it is important to measure the wake-up probability as a function of distance. We performed field tests of the WISP-Motes in a large hall, which is similar to an outdoor environment. We raised both the WISP and the reader's antenna off the ground to reduce multipath fading. We enabled the interrupt of the WISPMote periodically, and we counted the number of times the WISP-Mote can be successfully woken up as a function of distance. The test results, which determine the wake-up probability, are shown in Fig. 2.

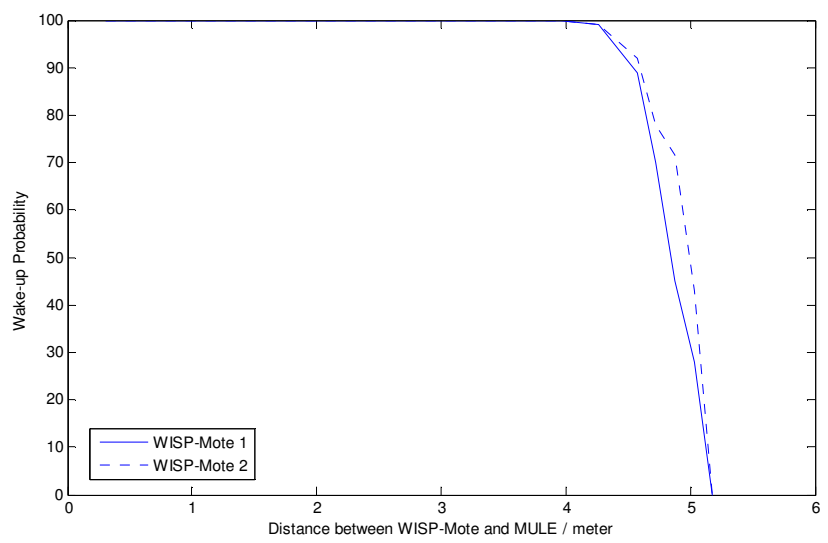

Figure 2. Wake-up probability of the WISP-Motes.

As seen in Fig. 2, the wake-up probability starts to decrease after $4 \mathrm{~m}$ and sharply drops down to 0 beyond $5 \mathrm{~m}$. In our simulations, we use a conservative value of $4 \mathrm{~m}$.

\section{Energy Consumption Measurements}

The major advantage of passive RFID wake-up is to reduce the energy waste of a sensor node and enhance its energy efficiency. The Tmote Sky datasheet [15] provides the current consumptions in typical operating conditions. We measured current consumption in booting and radio initiation, which is essential for the energy consumption analysis of RFID wake-up. The results are shown in Table II. Our measurements are consistent with those from the Tmote Sky datasheet. We can see that besides radio transmission and reception, node wakeup also consumes energy that cannot be ignored. This would support the need for an accurate energy analysis for the radio wake-up mechanism when characterizing a wake-up mote.

TABLE II. POWER CONSUMPTION MEASUREMENTS OF A T-MOTE SKY NODE

\begin{tabular}{|l|l|l|}
\hline \multicolumn{1}{|c|}{ Operation } & \multicolumn{1}{|c|}{$\begin{array}{c}\text { Average current } \\
\text { consumption }\end{array}$} & \multicolumn{1}{c|}{ Time } \\
\hline Wake-up and Radio Initiation & $10.4 \mathrm{~mA}$ & $5 \mathrm{~ms}$ \\
\hline Transmit 12 byte packet & $18.2 \mathrm{~mA}$ & $30 \mathrm{~ms}$ \\
\hline Receive and idle listening & $20.2 \mathrm{~mA}$ & \\
\hline Sleep & $0.2 \mathrm{~mA}$ & \\
\hline
\end{tabular}

\section{USE OF WISP-Motes IN A DATA MUlE SCENARIO}

The main advantage of the WISP-Motes is high energy efficiency through on-demand wake-up. However, a short wake-up range is achieved compared to the communication range. To evaluate the benefits of WISP-Motes, we consider a sparse delay-tolerant network of WISP-Motes with data MULEs that collect the sensor data. The MULE architecture provides connectivity for a sparse sensor network using singlehop communications. In this scenario, one or multiple mobile MULEs move throughout the network field collecting data from the sensor nodes. The MULEs are equipped with RFID readers and can wake up the WISP-Motes. Once a MULE is within wake-up range of a sensor node (within $4 \mathrm{~m}$ range for our simulations), the node is awakened and senses the channel if it has buffered data. If the channel is busy, the WISP-Mote 
will remain active and sense again in the next slot. Once the channel is free, the WISP-Mote will start transmitting its buffered data. If the WISP-Mote is not awakened by a MULE, the node remains asleep. In real scenarios, any moving agent, such as a person, an animal, or a vehicle, could act as a data MULE.

We compare the performance of the WISP-Mote network with a network of conventional sensor nodes that utilize duty cycling to save energy. In the latter case, the MULEs periodically send advertisement packets to declare their presence. Nodes periodically wake up, and if the node has buffered data, it will sense the channel. If a node receives an advertisement packet from a MULE, it responds by sending its buffered data. If the MULE is in communication with another node, the sensor node will stay active and sense the channel again in the next time slot. If there are no MULEs within the range of the sensor node, the sensor node returns to sleep until the next duty cycle wake-up period.

\section{A. Simulation Setup}

To simplify the simulations, we make the following assumptions:

- Propagation delay is ignored.

- We consider a simplified MAC layer and assume there are no collisions and no link failures. Once a node senses a free channel, it will send a packet with guaranteed arrival at the MULE.

- A MULE's appearance in one time slot is enough for a node to detect it and finish one packet transmission.

- MULEs have the ability to communicate directly to the data sink. Therefore, packet delay is counted from the time a packet is generated until the time it is delivered to a MULE.

- Whenever nodes receive MULE advertisement packets or sense the channel, they dissipate power (receive power) during the entire slot time.

- We ignore the energy cost for sensing activities as these will not impact the performance evaluation.

- The radio wake-up range is set to $4 \mathrm{~m}$. The mote's communication range is set to $40 \mathrm{~m}$ based on experiments described in [16] as well as our own field experiments.

In our network simulations, nodes are uniformly randomly deployed in a $200 \mathrm{~m} \times 200 \mathrm{~m}$ square region with a density of 0.001 nodes $/ \mathrm{m}^{2}$. MULEs begin with uniformly random locations, and they move at each time slot according to a Random Direction mobility model. Each MULE randomly selects a speed from $[5 \mathrm{~m} / \mathrm{s}, 15 \mathrm{~m} / \mathrm{s}]$ and a direction from $[0,2 \pi]$ and moves according to this speed and direction until it reaches the network boundary. Each node generates a packet every 10 minutes, i.e., with 0.1 packets $/ \mathrm{min}$. We compare the average packet delay and the energy consumed in 2 hours of operation for the WISP-Mote scenario and for the duty cycling scenario.
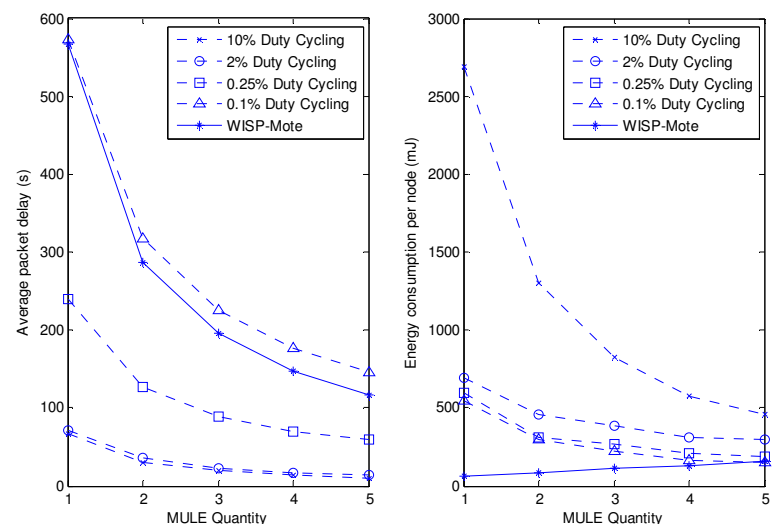

Figure 3. Packet delay and energy consumption comparisons as a function of the number of MULEs.

\section{B. Performance Results}

Fig. 3 shows the results of delay and energy consumption for $0.1 \%, 0.25 \%, 2 \%$ and $10 \%$ duty cycling and for the WISPMote. Compared to duty cycling, the WISP-Mote has to buffer data for a longer time until a MULE is within its wake-up range, which results in a high packet latency. On the other hand, in the duty cycling scenario, the lower the duty cycle value, the higher the probability of missing a MULE, since the nodes are in sleep mode longer. The resulting delay becomes large for very low duty cycle values (e.g., $0.1 \%$ ). Therefore, the delay performance of the WISP-Mote is worse than $10 \%, 2 \%$ and $0.25 \%$ duty cycling, but it achieves better delay than $0.1 \%$ duty cycling. The energy consumption values, provided in Fig. 3, show that the WISP-Mote uses much less energy than $0.1 \%$, $0.25 \%, 2 \%$ and $10 \%$ duty cycling, since the WISP-Mote does not waste energy in unnecessary wake-ups and idle listening.

Another important factor in the delay results is the number of MULEs in the area. More MULEs provide larger coverage per time unit and therefore decrease the packet latency. We can see from Fig. 3 that when the number of MULEs is increased from 1 to 5 , the average packet delay decreases by about $75 \%$ for both the WISP-Mote and the duty cycling scenarios. This provides a solution for applications with specific packet latency requirements at the cost of increasing the number of MULEs.
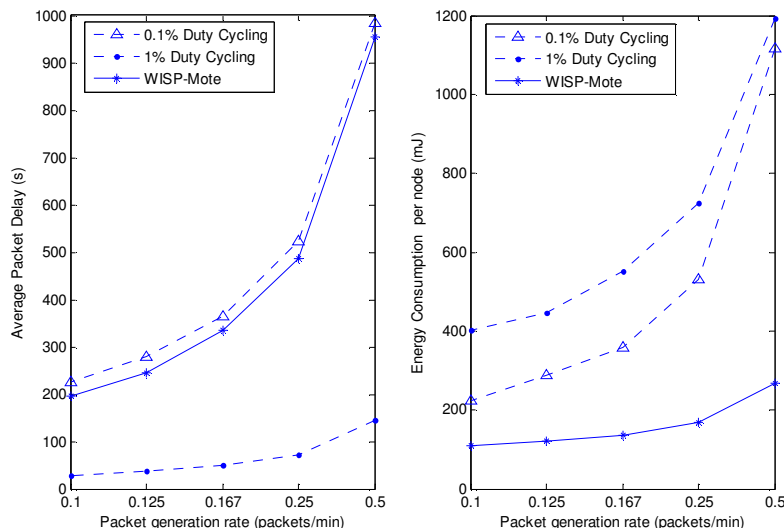

Figure 4. Packet delay and energy consumption comparisons as a function of packet generation rate. 
Fig. 4 shows the performance under various traffic loads for 3 MULEs. We assume only one node is allowed to transmit data to a certain MULE in one time slot and will consume energy in sensing again if the channel is busy. Therefore, increasing the traffic load leads to an increase in delay and energy consumption due to re-sensing the channel. The packet delay caused by re-sensing is not significant compared to the delay due to buffered data. We observe that when the packet generation rate increases from 0.1 packets $/ \mathrm{min}$ to 0.125 packets/min, the average packet delay of all three scenarios only increased slightly. However, when the packet generation rate increases further, the packet delays of $0.1 \%$ duty cycling and the WISP-Mote increase exponentially, due to accumulated data in the buffers. In the $1 \%$ duty cycling scenario, when packet generation rate is 0.5 packets/min, nodes are still able to deliver packets before new packets are generated. Therefore, the delay is still increased linearly. On the other hand, the energy consumptions in the duty cycling scenarios are dominated by re-sensing the channel when the packet generation rate is increased. The WISP-Mote scenario has less chance of re-sensing due to its limited wake-up range, which results in less energy consumption compared to the duty cycling scenarios.
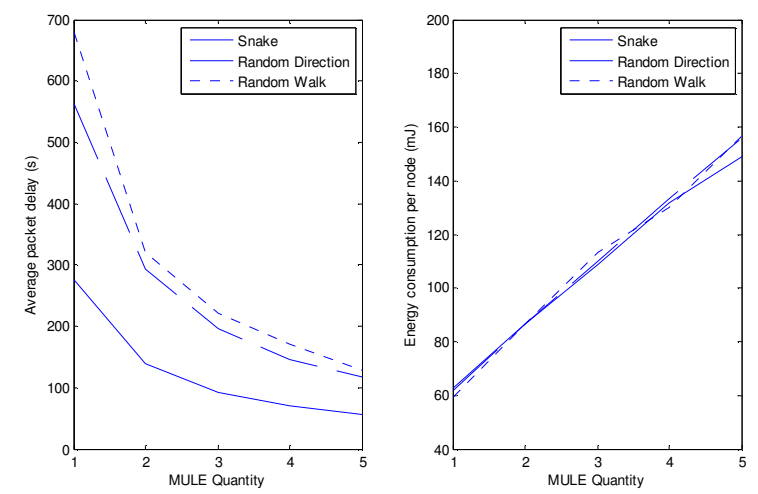

Figure 5. Mobility model comparisons.

The MULEs' mobility model also has an impact on the performance of the networks. We compare three different mobility models: Snake, Random Walk, and Random Direction. In the Snake algorithm, each MULE sweeps over the entire field by following a snake-shaped route with a constant velocity of $10 \mathrm{~m} / \mathrm{s}$. In the Random Walk algorithm, each MULE randomly selects a speed from $[5 \mathrm{~m} / \mathrm{s}, 15 \mathrm{~m} / \mathrm{s}$ ] and a direction from $[0,2 \pi]$ and moves according to this speed and direction for a random duration of between 1 and 100 time slots. The Random Direction mobility model is described in Section IV.A. The average velocities of the three models are the same. From Fig. 5, we can see that the Snake algorithm has the best performance in terms of packet delay while the energy consumption is virtually the same for all three mobility models. To reduce average packet delay, the MULEs could follow a scheduled route and go directly to the sensor nodes one by one.

\section{CONCLUSION}

In this paper, we present and characterize a physical implementation of a passive RFID wake-up device using existing hardware. In the Data MULE scenario, the benefit of our device in terms of reducing energy consumption is shown through simulation results. By trading off the extra hardware cost and increased packet latency, the lifetime of the entire network can be greatly extended. For a similar packet delay performance, a network utilizing WISP-Motes can save up to $89 \%$ of the energy consumption compared with $0.1 \%$ duty cycling for one MULE. To reduce the packet delay and improve the network robustness, multiple data MULEs can be deployed.

\section{ACKNOWLEDGEMENT}

The WISPs used in this research were donated by Intel as part of the WISP Challenge.

\section{REFERENCES}

[1] A. P. Sample, D. J. Yeager, P. S. Powledge, and J. R. Smith, "Design of an RFID-based battery-free programmable sensing platform," IEEE Transactions on Instrumentation and Measurement, Vol. 57, No. 11, Nov. 2008, pp. 2608-2615.

[2] J. Polastre, R. Szewczyk, and D. Culler, "Telos: Enabling Ultra-Low Power Wireless Research", The Fourth International Conference on Information Processing in Sensor Networks: Special track on Platform Tools and Design Methods for Network Embedded Sensors IPSN 2005: 364-369, April 25-27, 2005.

[3] R. C. Shah, S. Roy, S. Jain, and W. Brunette, "Data MULEs: modeling a three-tier architecture for sparse sensor networks", Proceedings of the First IEEE. 2003 IEEE International Workshop on (2003), pp. 30-41.

[4] N. Pletcher, S. Gambini, and J. Rabaey, "A $65 \mu \mathrm{W}, 1.9 \mathrm{GHz}$ RF to Digital Baseband Wakeup Receiver for Wireless Sensor Nodes," IEEE CICC 2007, San Jose, 16-19 Sept. 2007.

[5] M. J. Miller, and N. H. Vaidya, "Minimizing energy consumption in sensor networks using a wakeup radio", IEEE WCNC, 2004.

[6] P. Le-Huy and S. Roy, "Low-Power Wake-Up Radio for Wireless Sensor Networks," IEEE International Conference on Wireless and Mobile Computing, Networking and Communications, 2008.

[7] L. Gu and J. Stankovic, "Radio-Triggered Wake-up for Wireless Sensor Networks," Real-Time Systems archive. Volume 29, Issue 2-3, March 2005 .

[8] A. G. Ruzzelli, R Jurdak, and G. M. P. O'Hare, "On the RFID wake-up impulse for multi-hop sensor network", SenseID Workshop at ACM SenSys 2007, Sydney, Australia. November, 2007.

[9] R. Jurdak, A. G. Ruzzelli, and G. M. P. O'Hare, "Multi-hop RFID Wake-up Radio: Design, Evaluation and Energy Tradeoffs", Computer Communications and Networks, 2008. ICCCN '08.

[10] R. C. Shah, S. Roy, S. Jain, and W. Brunette, "Data MULEs: modeling a three-tier architecture for sparse sensor networks", Proceedings of the First IEEE. 2003 IEEE International Workshop on (2003), pp. 30-41.

[11] F. Zhang, B. Huang, Z. Xu, and L. Tu, "Delay Analysis of Single-hop Communication in Wireless Sensor Networks with Mobile Sink", Second International Multi-Symposiums on Computer and Computational Sciences, 2007. IMSCCS 2007.

[12] G. Anastasi, M. Conti, and M. Di Francesco, "Data collection in sensor networks with data mules: An integrated simulation analysis", IEEE Symposium on Computers and Communications, 2008. ISCC 2008.

[13] G. Lu, B. Krishnamachari, and C. S. Raghavendra, “An adaptive energyefficient and low-latency MAC for tree-based data gathering in sensor networks", Wirel. Commun. Mob. Comput. 2007, 7:863-875.

[14] http://wisp.wikispaces.com/

[15] Tmote Sky, http://sentilla.com/files/pdf/eol/tmote-sky-datasheet.pdf.

[16] M. Holland, R. Aures and W. Heinzelman, "Experimental Investigation of Radio Performance in Wireless Sensor Networks," IEEE SECON 2006 Poster Session, Sept. 2006. 